\title{
Cutting Time and Regrowth Age Affect the Quality of Elephant Grass Silage
}

\author{
Tâmara Chagas da Silveira
}

Universidade Federal de Viçosa, Department of Animal Science, Viçosa, Minas Gerais, Brazil, 36570-900. E-mail: tandacs@gmail.com

\section{Karina Guimarães Ribeiro (Corresponding Author)}

Universidade Federal de Viçosa, Department of Animal Science, Viçosa, Minas Gerais, Brazil, 36570-900. E-mail: karinaribeiro@ufv.br

\section{João Paulo Santos Roseira}

Universidade Federal de Viçosa, Department of Animal Science, Viçosa, Minas Gerais, Brazil, 36570-900. E-mail: jpr-santos@hotmail.com

\section{Wagner Sousa Alves}

Universidade Federal de Viçosa, Department of Animal Science, Viçosa, Minas Gerais, Brazil, 36570-900. E-mail: wagner.sousa@ufv.br

\section{Albert Jose dos Anjos}

Universidade Federal de Viçosa, Department of Animal Science, Viçosa, Minas Gerais, Brazil, 36570-900. E-mail: albert@ zootecnista.com.br

\section{Danielle Nascimento Coutinho}

Universidade Federal de Viçosa, Department of Animal Science, Viçosa, Minas Gerais, Brazil, 36570-900. E-mail: daniellencout@gmail.com

\section{Cássia Aparecida Soares Freitas}

Universidade Federal de Viçosa, Department of Animal Science, Viçosa, Minas Gerais, Brazil, 36570-900. E-mail: cassia.freitas@ufv.br 
Odilon Gomes Pereira

Universidade Federal de Viçosa, Department of Animal Science, Viçosa, Minas Gerais, Brazil, 36570-900. E-mail: odilon@ufv.br

Received: April 27, 2021 Accepted: June 6, $2021 \quad$ Published: June 16, 2021

doi:10.5296/jas.v9i3.18566

URL: https://doi.org/10.5296/jas.v9i3.18566

\begin{abstract}
The objective of this study was to evaluate the effects of regrowth age (RA) and cutting time (CT) of elephant grass (Cenchrus purpureus cv. Cameroon) on the fermentation profile, microorganism population, and nutritive value of the silage in two simultaneous trials at different sites. A $2 \times 2$ factorial scheme with two CTs (08:00 and 14:00) and two RAs (8 and 16 weeks) at ensilage was used in a completely randomized design, with four replicates in each trial, totaling 16 experimental units per trial. Results showed that ammonia nitrogen to total nitrogen content $\left(\mathrm{N}-\mathrm{NH}_{3} / \mathrm{TN}\right)$ ranged from $71.8\left(14: 00 ; 16\right.$ weeks) to $137.0 \mathrm{~g} \mathrm{~kg}^{-1}$ (14:00; 8 weeks). Elephant grass silage harvested at 14:00 at 8 weeks had the lowest $\mathrm{pH}$ (3.53) and highest lactic acid content $\left(39.7 \mathrm{~g} \mathrm{~kg}^{-1}\right.$ dry matter). The in vitro dry matter digestibility (IVDMD) was higher $(P<0.05)$ in the silage of plants harvested at 8 weeks than in the silage harvested at 16 weeks (59.9 vs. $46.8 \%$, respectively). In trial 2 , elephant grass silage harvested at 14:00 had lower $\mathrm{pH}$ and acetic acid content. The IVDMD was higher in elephant grass silage harvested at 14:00, with values of $65.3 \%$ and $56.2 \%$ at 8 and 16 weeks, respectively. We recommend elephant grass harvested at 8 weeks of regrowth and cut at 14:00 to produce silage with better nutritive value and fermentative profile.
\end{abstract}

Keywords: fermentative profile, microbial population, nutritive value

\title{
1. Introduction
}

Elephant grass [Cenchrus purpureus (Schumach.) Morrone; Basionym: Pennisetum purpureum Schumach.] is among the most popular forage grasses for silage production in tropical regions because of its high productivity and good nutritional value (Pereira et al., 2017). However, tropical grasses are characterized by high moisture content and low soluble carbohydrate content (Pholsen et al., 2016), which can negatively affect forage fermentation in the silo.

The concentration of soluble carbohydrates in plants is fundamental to the fermentation process. At low concentrations of soluble carbohydrates, the amount of lactic acid produced by bacteria is insufficient to reduce $\mathrm{pH}$ and inhibit the growth of deleterious microorganisms, resulting in low-quality silage (Pholsen et al., 2016; Wang et al., 2019).

The concentration of soluble carbohydrates in plants increases during the day when their photosynthetic production exceeds use (Bernardes et al., 2018). Accordingly, higher 
concentrations of soluble carbohydrates in forage plants harvested after 11:00 am were observed by some authors in elephant grass cv. Napier (Cenchrus purpureus cv. Napier; De Oliveira et al., 2014; Guo et al., 2015), ryegrass (Lolium multiflorum Lam.; Guo et al., 2014), alfalfa (Medicago sativa L.; Tremblay et al., 2014), Marandu palisade grass (Urochloa brizantha cv. Marandu; De Oliveira et al., 2018), and mixed birdsfoot trefoil (Lotus corniculatus), and timothy grass (Phleum pratense; Silva et al., 2020). Due to the higher soluble carbohydrate concentration in plants harvested at a later time of the day, there is greater substrate availability for lactic acid bacteria during the fermentation phase, resulting in higher lactic acid production and a rapid reduction in $\mathrm{pH}$. Therefore, the harvest time of forage plants could be used as a management strategy to produce better quality silage.

The nutritional value of elephant grass is also significantly influenced by maturity (Haryani et al., 2018). Zailan et al. (2016) evaluated four elephant grass cultivars harvested at 4, 6, and 8 weeks and observed an increase in the dry matter (DM), neutral detergent fiber (NDF) content, and acid detergent fiber (ADF) content, and a decrease in the crude protein (CP) content and in vitro DM digestibility, with an increase in harvest age. In a study of elephant grass silage cv. BRS Capiaçu harvested at 50-110 days of growth, Lopes et al. (2021) also found a decrease in the $\mathrm{CP}$ content and an increase in the NDF content, as well as a decrease in silage $\mathrm{pH}$.

Evidence in literature demonstrates the effects of cutting time (CT) and regrowth age (RA) of elephant grass on plant nutritive value and the fermentative profile of the silages produced. However, there are no studies on the effects of combining these two factors on the nutritive value and fermentative profile of elephant grass silage. Therefore, we hypothesized that CT and RA, or their interaction affects the nutritive value, especially soluble carbohydrate content, and fermentation of elephant grass during ensiling. We aimed to identify the best combination for silage production without the need for wilting and additive use.

\section{Material and Methods}

\subsection{Test Sites, Soil, and Climatic Conditions}

Two trials (Trials 1 and 2) were conducted simultaneously at two different sites based on soil chemical characteristics at the Animal Science Department of the Federal University of Viçosa (DZO), UFV, at the Viçosa Campus, from September 2015 to April 2016. The municipality of Viçosa $\left(20^{\circ} 45^{\prime} 20^{\prime \prime} \mathrm{S}, 42^{\circ} 52^{\prime} 40^{\prime \prime \prime} \mathrm{W}\right.$; altitude, $\left.657 \mathrm{~m}\right)$ is in Zona da Mata, in the state of Minas Gerais. The climate is of the Cwa type according to the Köppen classification, with two defined seasons: dry, from May to October, and wet, from November to April. The soil is classified as eutrophic red-yellow Argisol clay at both sites (Santos et al., 2018).

Organic fertilizer in the form of dry bovine manure was applied to the elephant grass in site 1 and as bovine manure water $(1: 1)$ in site 2 . The chemical characteristics of soil samples from the two sites are listed in Table 1. 


\section{Ml Macrothink}

Journal of Agricultural Studies

ISSN 2166-0379

2021, Vol. 9, No. 3

Table 1. Chemical analysis of soil samples collected in the $0-20 \mathrm{~cm}$ layer, in the two experimental sites

\begin{tabular}{|c|c|c|c|c|c|c|c|c|c|c|}
\hline & $\begin{array}{c}\mathrm{pH} \\
\mathrm{H}_{2} \mathrm{O}\end{array}$ & $\begin{array}{c}\mathrm{P} \\
\mathrm{mg} / \\
\mathrm{dm}^{3} \\
\end{array}$ & $\begin{array}{c}\mathrm{K} \\
\mathrm{mg} / \mathrm{dm}^{3}\end{array}$ & $\begin{array}{c}\mathrm{Ca}^{2+} \\
\mathrm{cmolc} / \\
\mathrm{dm}^{3}\end{array}$ & $\begin{array}{c}\mathrm{Mg}^{2+} \\
\mathrm{cmolc}^{2} \\
\mathrm{dm}^{3} \\
\end{array}$ & $\begin{array}{c}\mathrm{Al}^{3+} \\
\mathrm{cmolc}^{+} / \\
\mathrm{dm}^{3} \\
\end{array}$ & $\begin{array}{c}\mathrm{SB} \\
\mathrm{cmolc} / \\
\mathrm{dm}^{3}\end{array}$ & $\begin{array}{c}\mathrm{t} \\
\mathrm{cmolc} / \\
\mathrm{dm}^{3}\end{array}$ & $\begin{array}{c}\mathrm{T} \\
\mathrm{cmolc} / \\
\mathrm{dm}^{3} \\
\end{array}$ & $\begin{array}{l}\mathrm{V} \\
\%\end{array}$ \\
\hline $\begin{array}{l}\text { Site } \\
1\end{array}$ & 5.60 & 19.9 & 73 & 5.78 & 1.11 & 0.00 & 7.08 & 7.08 & 11.58 & 61.1 \\
\hline $\begin{array}{l}\text { Site } \\
2\end{array}$ & 6.18 & 359 & 315 & 5.28 & 1.81 & 0.00 & 7.90 & 7.90 & 11.00 & 71.8 \\
\hline
\end{tabular}

$\mathrm{pH}$ (in water, $\mathrm{KCl}$ e $\mathrm{CaCl}$ - Ratio 1:2.5); $\mathrm{SB}$ (sum of exchangeable bases); $\mathrm{t}$ (Capacity of effective cation exchange); $\mathrm{T}$ (Cation exchange capacity at $\mathrm{pH}$ 7.0); V (Base saturation index).

\subsection{Ensiling and Experimental Design}

The treatments of both trials were arranged in a $2 \times 2$ factorial scheme, with two CTs (08:00 and 14:00) and two RAs (8 and 16 weeks) of elephant grass (Cenchrus purpureus Schum. cv. Cameroon) during ensiling, in a completely randomized design, with four replicates in each trial, totaling 16 experimental units per trial.

Prior to harvesting the elephant grass for ensiling, two uniform cuts were made at both sites such that at the time of ensiling (01/27/2016), the plants had reached the two recommended RAs. The first cut was made in September 2015 so that the elephant grass would have had 16 weeks of regrowth at the time of cutting for silage. The second cut was made in November 2015 so that at the time of ensiling, the elephant grass would have had 8 weeks of regrowth.

During the regrowth of plants harvested at 16 weeks, the accumulated rainfall was $916.6 \mathrm{~mm}$, and the maximum, average, and minimum temperatures were 30,23 , and $18{ }^{\circ} \mathrm{C}$, respectively (INMET, 2016). During the regrowth of plants harvested at 8 weeks, the accumulated rainfall was $652.2 \mathrm{~mm}$, and the maximum, average, and minimum temperatures were 29, 23, and $19{ }^{\circ} \mathrm{C}$, respectively (INMET, 2016). Elephant grass was harvested simultaneously, $5 \mathrm{~cm}$ from the ground at both sites, using a Stihl FR220R costal machine (STIHL Brasil, São Leopoldo, Brazil), and ensiled separately by site.

The forage was chopped using a stationary forage machine and packed in piles of approximately $8 \mathrm{~kg}$ to fill each bucket. A sample from each pile was collected to assess the buffering capacity, $\mathrm{pH}$, microorganism population, and chemical composition of elephant grass before ensiling.

To make the silage, $12 \mathrm{~kg}$ plastic buckets were used as experimental silos. After weighing the buckets with lids, they were filled, and the forage was compacted with the aid of wooden sockets. Silage densities varied between 141 and $182 \mathrm{~kg} / \mathrm{m}^{3} \mathrm{DM}$, owing to the different DM content of the samples. The silos were then sealed, weighed, and stored in a covered area at an average temperature of $23{ }^{\circ} \mathrm{C}$ for $60 \mathrm{~d}$.

The experimental silos were then weighed and opened, and the silage within each silo was homogenized. An aliquot of the material was removed and divided into two sub-samples; the first (25 g) was used to quantify the lactic acid bacteria (LAB) population, enterobacteria, fungi (molds and yeasts), and the fermentative profile ( $\mathrm{pH}$, organic acids, ammonia, and 
ethanol), and the second sample (300 g) was used for chemical composition analysis.

\subsection{Quantification of Microbial Populations}

To quantify the microorganism population, an aqueous extract was prepared containing $25 \mathrm{~g}$ of plant or silage sample, which was homogenized together with $225 \mathrm{~mL}$ of buffer solution (Ringer Solution ${ }^{\circledR}$; Oxoid, Hampshire, United Kingdom) for $1 \mathrm{~min}$ in a blender to obtain a $10^{-1}$ dilution (Kung Jr., 1996). Subsequently, $10 \mathrm{~mL}$ of the aqueous extract was subjected to serial dilutions ranging from $10^{-2}$ to $10^{-7}$. The microorganisms were cultured in sterile Petri dishes in appropriate culture media for different microorganism groups. MRS agar (Merck KGaA, Darmstadt, Germany) was used for LAB, while Violet Red Bile Agar (Oxoid) and potato dextrose agar (PDA; Difco, Sao Paulo, Brazil) supplemented with $1.5 \%$ tartaric acid at $10 \%$ were used for enterobacteria and molds and yeasts, respectively, using the pour-plate plating technique. The plates were incubated in a TE-391 Biochemical Oxygen Demand incubator (Tecnal, Piracicaba, Brazil) at temperatures and periods determined for each group of microorganisms: enterobacteria, $37{ }^{\circ} \mathrm{C}$ for $24 \mathrm{~h}$; LAB, $37{ }^{\circ} \mathrm{C}$ for $48 \mathrm{~h}$; yeasts and molds at $25{ }^{\circ} \mathrm{C}$ for 72 and $120 \mathrm{~h}$, respectively. At the end of the incubation period, counting was conducted using a manual colony counter (Model CP 608; Phoenix Luferco, Araraquara, Brazil). Plates that had 30-300 colony forming units (CFU) were counted. For data evaluation and interpretation, the results were converted to a logarithmic basis $(\log \mathrm{CFU} / \mathrm{g}$ ) (Kung Jr. et al., 2003).

\subsection{Fermentative Profile and Chemical Composition}

A sample of approximately $150 \mathrm{~g}$ of chopped elephant grass was collected before ensiling to analyze soluble carbohydrate content (Nelson 1944) and buffer capacity (Playne and McDonald 1966).

The fermentative capacity (FC) of forages before ensiling was calculated according to the equation proposed by Kaiser et al. (2002):

$\mathrm{FC}=\mathrm{DM}+8 \times(\mathrm{SC} / \mathrm{BC})$,

where $\mathrm{DM}$ is dry matter (\%), $\mathrm{SC}$ is soluble carbohydrates (\%), and $\mathrm{BC}$ is buffering capacity (e.mg of $\mathrm{NaOH} / 100 \mathrm{~g} \mathrm{DM})$.

The $\mathrm{pH}$ of the initial aqueous extract ( $25 \mathrm{~g}$ silage/225 $\mathrm{mL}$ of Ringer Solution) of the samples was measured using a digital potentiometer (Tecnal), as described by Kung Jr. (1996).

A $10 \mathrm{~mL}$ aliquot was removed from the aqueous extract and placed in Falcon tubes containing a sulfuric acid solution (50\%) and frozen for further analysis of organic acids (lactic, acetic, propionic, and butyric acids) and ethanol. Organic acid and ethanol analyses were conducted using a high-performance liquid chromatograph (HPLC; Accella PDA model; Thermo Fisher Scientific, Hemel Hempstead, UK) coupled with an ultraviolet (UV) detector at $210 \mathrm{~nm}$ wavelength, as described by Siegfried et al. (1984). The ammonia nitrogen to total nitrogen content $\left(\mathrm{N}-\mathrm{NH}_{3} / \mathrm{TN}\right)$ was also quantified according to the methodology described by Okuda et al. (1965). 


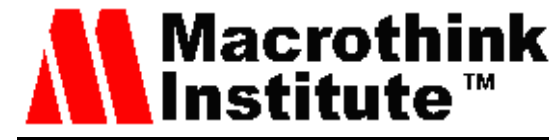

To evaluate the chemical composition of the samples, partial moisture removal was carried out in an oven with forced-air ventilation at $55^{\circ} \mathrm{C}$ until a constant weight was achieved. Subsequently, the samples were processed in a Model 4 Wiley Mill (Thomas Scientific, Swedesboro, NJ, USA) with a $1 \mathrm{~mm}$ sieve. The DM, CP, neutral detergent fiber corrected for ash and protein (NDFap), ADF, mineral matter, neutral detergent insoluble nitrogen (NDIN), and acid detergent insoluble nitrogen (ADIN) contents were determined according to the methodologies described by Detmann et al. (2012).

For the in vitro dry matter digestibility (IVDMD) test, silage samples $(0.5 \mathrm{~g})$ were placed in ANKOM F57 ${ }^{\circledR}$ filter bags (Ankom Technology, Macedon, NY, USA) and incubated in an ANKOM Daisy Incubator for $48 \mathrm{~h}$ in jars containing buffer solution and ruminal fluid. The ruminal fluid was collected from fistulated cattle as described by Holden (1999).

The quality of all elephant grass silages was estimated using "Flieg's point," calculated using the equation proposed by Kilic (1986), cited by Moselhy et al. (2015):

Flieg's point $=220+(2 \times \% \mathrm{DM}-15)-40 \times \mathrm{pH}$

According to this classification, the silage quality was considered "very bad" at an index <20; "bad" at an index between 21 and 40; "average" at an index between 41 and 60; "good" at an index between 61 and 80; and "very good" between 81 and 100 .

\subsection{Statistical Analysis}

Data were analyzed according to a completely randomized design in a $2 \times 2$ factorial, using PROC MIXED of SAS version 9.4 (SAS Institute, Inc., Cary, NC, USA). The RAs, CTs, and interaction between these factors were considered fixed effects in the model:

$\mathrm{Y}_{\mathrm{ijk}}=\mu+\mathrm{RA}_{\mathrm{i}}+\mathrm{H}_{\mathrm{j}}+(\mathrm{RA} \times \mathrm{H})_{\mathrm{ij}}+\mathrm{e}_{\mathrm{ijk}}$,

where

$Y_{i j k}$ is the dependent variable, $\mu$ is the general mean, $R A_{i}$ is the effect of $R A, H_{j}$ is the effect of $\mathrm{CT},(\mathrm{RA} \times \mathrm{H})_{\mathrm{ij}}$ is the interaction between factors, and $\mathrm{e}_{\mathrm{ijk}}$ is the random error, assuming a normal independent distribution, $(0 ; \sigma 2 \varepsilon)$. The treatment means were subjected to analysis of variance and comparison using the F-test, adopting 0.05 as the critical level of probability for type I error.

\section{Results}

3.1 Chemical Composition, Buffer Capacity, pH, and Epiphytic Microflora of Elephant Grass Before Silage

Tables 2 (Trial 1) and 5 (Trial 2) show the results for chemical composition, buffer capacity, $\mathrm{pH}$, and epiphytic microflora of elephant grass harvested at different CT and RA. The highest concentrations of soluble carbohydrates were observed in elephant grass harvested at 14:00 than at 08:00 at both RAs. The highest concentrations of soluble carbohydrates were observed for grass harvested at 8 weeks than at 16 weeks at both CTs. 
Table 2. Chemical composition, buffering capacity, $\mathrm{pH}$, and epiphytic microflora of elephant grass harvested at different cutting times (CT) and regrowth ages (RA)

\begin{tabular}{lcccc}
\hline \multirow{2}{*}{\multicolumn{1}{c}{ Item }} & \multicolumn{2}{c}{8 weeks } & \multicolumn{2}{c}{16 weeks } \\
\cline { 2 - 5 } & $08: 00 \mathrm{~h}$ & $14: 00 \mathrm{~h}$ & $08: 00 \mathrm{~h}$ & $14: 00 \mathrm{~h}$ \\
\hline $\mathrm{DM}^{1}\left(\mathrm{~g} \mathrm{~kg}^{-1} \mathrm{FM}^{10}\right)$ & 132.5 & 154.6 & 238.3 & 297.8 \\
$\mathrm{WSC}^{2}\left(\mathrm{~g} \mathrm{~kg}^{-1} \mathrm{DM}\right)$ & 66.10 & 87.10 & 47.80 & 59.10 \\
$\mathrm{CP}^{3}\left(\mathrm{~g} \mathrm{~kg}^{-1} \mathrm{DM}\right)$ & 89.90 & 73.80 & 47.00 & 41.80 \\
$\mathrm{NDFap}^{4}\left(\mathrm{~g} \mathrm{~kg}^{-1} \mathrm{DM}\right)$ & 723.20 & 662.80 & 769.10 & 746.90 \\
$\mathrm{ADF}^{5}\left(\mathrm{~g} \mathrm{~kg}^{-1} \mathrm{DM}\right)$ & 496.00 & 489.20 & 538.30 & 541.30 \\
$\mathrm{NDIN}^{6}(\% \mathrm{NT})$ & 27.55 & 30.62 & 32.33 & 31.74 \\
$\mathrm{ADIN}^{7}(\% \mathrm{NT})$ & 7.31 & 7.85 & 12.66 & 13.94 \\
$\mathrm{BC}^{8}(\mathrm{meq} \mathrm{de} \mathrm{NaOH} / 100 \mathrm{~g} \mathrm{DM})$ & 10.80 & 9.05 & 6.15 & 5.10 \\
$\mathrm{pH}^{\mathrm{L}}$ & 6.11 & 6.03 & 6.04 & 5.99 \\
$\mathrm{LAB}^{9}(\log \mathrm{ufc} / \mathrm{g} \mathrm{FM})$ & 6.15 & 6.12 & 7.02 & 6.18 \\
Enterobacteria $(\log$ ufc/g FM$)$ & 6.24 & 6.67 & 5.87 & 6.15 \\
Mold and yeast $(\log$ ufc/g FM) & 5.07 & 5.10 & 6.07 & 5.50 \\
\hline
\end{tabular}

${ }^{1} \mathrm{DM}$, dry matter; $\mathrm{WSC}^{2}$, water-soluble carbohydrate ${ }^{2} ; \mathrm{CP}^{3}$, crude protein; ${ }^{4} \mathrm{NDFap}$ neutral detergent fiber corrected for ash and protein; ${ }^{5} \mathrm{ADF}$, acid detergent fiber; ${ }^{6} \mathrm{NDIN}$, neutral detergent insoluble nitrogen; ${ }^{7} \mathrm{ADIN}$, acid detergent insoluble nitrogen; ${ }^{8} \mathrm{BC}$, buffering capacity. ${ }^{9} \mathrm{LAB}$, lactic acid bacteria; ${ }^{10} \mathrm{FM}$, fresh matter.

\subsection{Fermentative Profile and Microbial Population of Elephant Grass Silages}

\subsubsection{Trial 1}

The results for Trial 1 are presented in Table 3. $\mathrm{pH}$, lactic acid, and ethanol were significantly affected $(P<0.05)$ by $\mathrm{CT}$ and RA. The silages produced with elephant grass harvested at 14:00 or 8 weeks of regrowth showed the lowest $\mathrm{pH}$ values $(P<0.05)$ and the highest lactic acid and ethanol levels $(P<0.05)$.

Table 3. Fermentation profile and microbial population of elephant grass silages harvested at different cutting times (CT) and regrowth ages (RA)

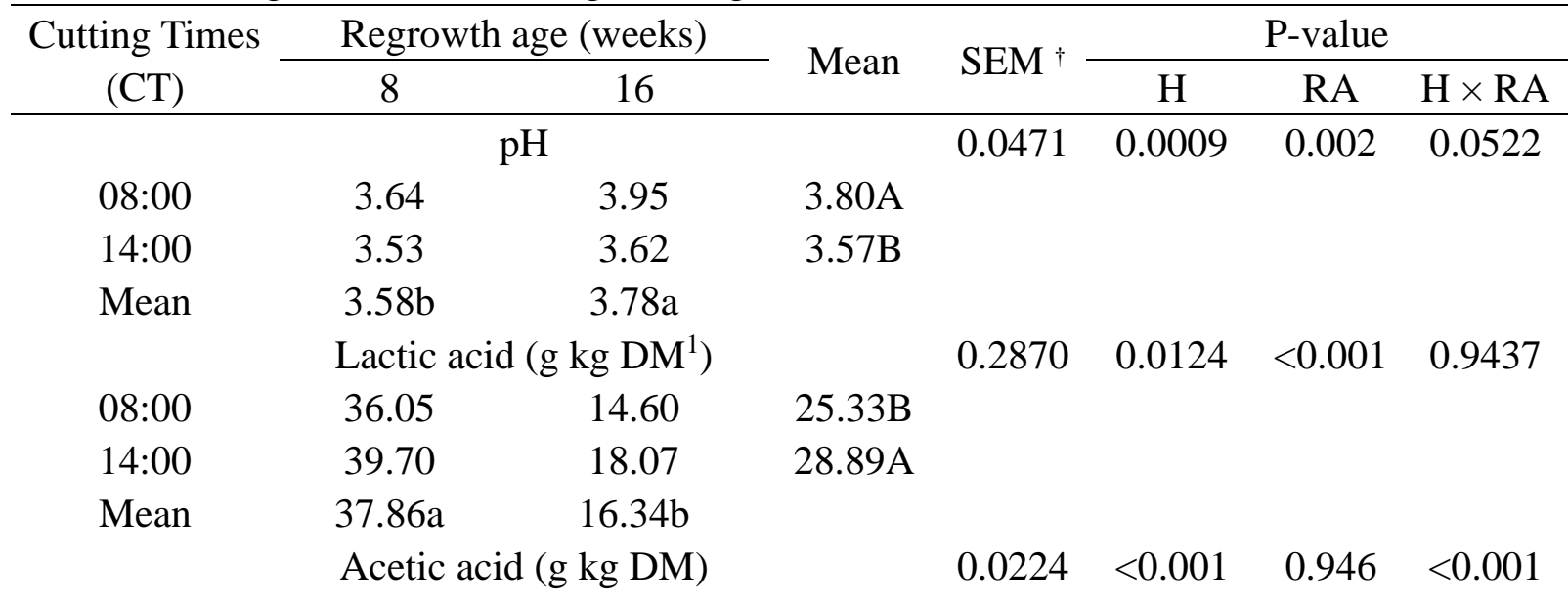




$\begin{array}{cccc}08: 00 & 5.37 \mathrm{Ab} & 6.60 \mathrm{Aa} & 5.99 \\ 14: 00 & 5.57 \mathrm{Aa} & 4.32 \mathrm{Bb} & 4.95 \\ \text { Mean } & 5.57 & 5.46 & \end{array}$

Propionic acid (g kg DM)

$\begin{array}{cccc}08: 00 & 5.00 \mathrm{Aa} & 1.72 \mathrm{Bb} & 3.36 \\ 14: 00 & 4.60 \mathrm{Aa} & 2.27 \mathrm{Ab} & 3.43 \\ \text { Mean } & 4.80 & 2.00 & \end{array}$

$$
\begin{array}{llll}
0.0372 & 0.6008 & <0.001 & 0.0052
\end{array}
$$

Butyric acid (g kg DM)

$\begin{array}{cccc}08: 00 & 0.62 \mathrm{Ab} & 1.47 \mathrm{Aa} & 1.05 \\ 14: 00 & 0.82 \mathrm{Aa} & 0.67 \mathrm{Ba} & 0.75 \\ \text { Mean } & 0.72 & 1.07 & \end{array}$

Ethanol (g kg DM)

08:00

14:00

Mean

08:00

14:00

Mean

08:00

14:00

Mean

08:00

14:00

Mean

08:00
14:00
Mean

08:00

14:00

4.82

10.80

7.47

$8.19 \mathrm{a}$

$6.15 b$

$\mathrm{NH}_{3}-\mathrm{N}(\mathrm{g} \mathrm{kg} \mathrm{TN})$

$\begin{array}{cc}101.2 \mathrm{Ba} & 90.8 \mathrm{Aa} \\ 137.0 \mathrm{Aa} & 71.8 \mathrm{Bb} \\ 119.1 & 81.3\end{array}$

$\mathrm{LAB}^{3}\left(\log\right.$ cfu g FM $\left.{ }^{5}\right)$.

Enterobacter (log cfu g FM)

$\begin{array}{ll}\mathrm{ND}^{4} & \mathrm{ND} \\ \mathrm{ND} & \mathrm{ND} \\ \mathrm{ND} & \mathrm{ND}\end{array}$

Mold and yeast (log cfu $\mathrm{g}$

$$
\text { FM) }
$$

1.50

2.89

Flieg's Point

$\begin{array}{ccc}6.58 \mathrm{Bb} & 8.25 \mathrm{Aa} & 7.41 \\ 7.05 \mathrm{Ab} & 8.06 \mathrm{Ba} & 7.55 \\ 6.81 & 8.15 & \end{array}$

Mean

96.0

104.4

$$
\begin{array}{llll}
0.1799 & 0.0391<0.001<0.001
\end{array}
$$

$$
\begin{array}{llll}
0.0125 & 0.1585 & 0.104 & 0.0276
\end{array}
$$

$0.0653<0.001 \quad 0.005 \quad 0.0526$

$\begin{array}{llll}0.6481 & 0.1039<0.001<0.001\end{array}$ 7.41

ND

ND

$\begin{array}{lll}\mathrm{ND} & 2.91 \mathrm{~A} & 1.45 \\ & 2.87 \mathrm{Aa} & 2.94\end{array}$

$0.3293<0.001<0.001<0.001$ 2.94

† SEM mean standard error; ${ }^{1} \mathrm{DM}$, dry matter; ${ }^{2} \mathrm{NH}_{3} / \mathrm{TN}$, ammoniacal nitrogen in relation to total nitrogen; ${ }^{3} \mathrm{LAB}$, lactic acid bacteria, ${ }^{4} \mathrm{ND}$, not detected (count less than dilution $10^{-1}$ ); ${ }^{5} \mathrm{FM}$, fresh matter. Means followed by the same capital letter, in the column, and lower case, in the line, do not differ $(\mathrm{P}>0.05)$ from each other by the $\mathrm{F}$ test. 
The RA $\times$ CT interaction significantly affected $(P<0.05)$ the levels of acetic, propionic, and butyric acids, ammonia nitrogen, and populations of LAB and fungi. The silages produced with 16 week plants harvested at 14:00 had a lower concentration of acetic acid than those harvested at 8:00. Silages produced at 16 weeks showed lower propionic acid concentrations than those produced at 8 weeks at both CTs. The butyric acid concentration was higher in silages from plants harvested at 16 weeks and 08:00. Lower concentrations of ammoniacal nitrogen $\left(\mathrm{NH}_{3}-\mathrm{N} / \mathrm{TN}\right)$ were obtained in silage produced with 16 week plants harvested at 14:00.

Larger LAB populations were observed in elephant grass silages at 16 weeks compared with plant silages at 8 weeks at both CTs. Elephant grass silages harvested at 8 weeks at 08:00 contained no fungi (molds and yeasts). No enterobacteria populations were observed in the silages at the evaluated CTs and RAs.

Flieg's point values were significantly affected $(P<0.05)$ by the $\mathrm{H} \times \mathrm{RA}$ interaction (Table 2$)$. Silage production using elephant grass harvested at 14:00 at both RAs resulted in higher Flieg's point values than elephant grass harvested at 08:00, and elephant grass silage harvested at a regrowth age of 16 weeks showed higher Flieg's Point values, compared with that harvested at 8 weeks at both CTs.

\subsubsection{Trial 2}

Trial 2 results are presented in Table 6 . There was a significant RA $\times$ CT interaction $(P<0.05)$ effect on the variables $\mathrm{pH}$, lactic, acetic, propionic, and butyric acids, and ammoniacal nitrogen. Higher $\mathrm{pH}$ value $(P<0.05)$ was observed when the ensiled plants were harvested at 08:00 than 14:00 with 8 weeks of regrowth.

Higher lactic acid levels $(P<0.05)$ were obtained in the silage of elephant grass harvested at 8 weeks compared with elephant grass harvested at 16 weeks of regrowth at the two CTs. Acetic acid production was lower $(P<0.05)$ in silage produced from elephant grass harvested at 14:00 than the silage from elephant grass harvested at 08:00, during the two RAs. Higher contents of acetic and butyric acids $(P<0.05)$ and ammoniacal nitrogen $(P<0.05)$ were observed in the silage of elephant grass harvested at 8 weeks at 08:00.

Ethanol content and LAB population were affected $(P>0.05)$ only by RA. Higher $(P<0.05)$ LAB populations and lower $(\mathrm{P}<0.05)$ ethanol concentrations were obtained in silage produced from plants harvested at 16 weeks than from that harvested at 8 weeks. Enterobacteria and fungi (molds and yeasts) populations were not observed in these silages.

The Flieg's point values were affected $(P<0.05)$ by the $\mathrm{CT} \times \mathrm{RA}$ interaction (Table 5), being higher in silages produced at 14:00 than those produced at 08:00 at the two RAs. Whereas silage of elephant grass harvested at 16 weeks presented higher Flieg's point values than that harvested at 8 weeks at both CTs. 


\subsection{Nutritional Value of Elephant Grass Silages}

\subsubsection{Trial 1}

Trial 1 results are listed in Table 4. DM and CP levels were significantly affected $(P<0.05)$ by the CT $\times$ RA interaction. Silage produced from elephant grass harvested at 14:00 showed higher DM levels and lower CP levels than silages produced from elephant grass harvested at 08:00 at both RAs. Silage produced from elephant grass harvested at 16 weeks of regrowth had higher DM levels $(P<0.05)$ and lower CP levels than silage from elephant grass harvested at 8 weeks of regrowth at both CTs. The levels of NDFap, ADF, and the IVDMD coefficients varied significantly $(P<0.05)$ with RA, while NDIN and ADIN levels varied with time and age. The levels of NDF, ADF, NDIN, and ADIN were lower for silage from plants harvested at 8 weeks; consequently, the IVDMD of this silage was higher. Higher levels of NDIN and ADIN were observed in the silage of elephant grass harvested at 14:00.

Table 4. Nutritive value of elephant grass silages harvested at different cutting times (CT) and regrowth ages (RA)

\begin{tabular}{|c|c|c|c|c|c|c|c|}
\hline \multirow{2}{*}{$\begin{array}{c}\text { Cutting Time } \\
\text { (CT) }\end{array}$} & \multicolumn{2}{|c|}{ Regrowth age (weeks) } & \multirow{2}{*}{ Mean } & \multirow{2}{*}{$\mathrm{SEM}^{\dagger}$} & \multicolumn{3}{|c|}{ P-value } \\
\hline & 8 & 16 & & & $\mathrm{H}$ & RA & $\mathrm{H} \times \mathrm{RA}$ \\
\hline \multicolumn{4}{|c|}{$\overline{\mathrm{DM}^{1}\left(\mathrm{~g} \mathrm{~kg} \mathrm{FM}^{2}\right)}$} & 1.6184 & $<0.001$ & $<0.001$ & $<0.001$ \\
\hline 08:00 & $144.4 \mathrm{Bb}$ & $246.0 \mathrm{Ba}$ & 195.2 & & & & \\
\hline $14: 00$ & $162.9 \mathrm{Ab}$ & $298.8 \mathrm{Aa}$ & 230.8 & & & & \\
\hline Mean & 153.7 & 272.4 & & & & & \\
\hline \multicolumn{4}{|c|}{$\mathrm{CP}^{3}(\mathrm{~g} \mathrm{~kg} \mathrm{DM})$} & 0.4779 & $<0.001$ & $<0.001$ & 0.0003 \\
\hline 08:00 & 84.92Aa & $44.87 \mathrm{Ab}$ & 64.90 & & & & \\
\hline $14: 00$ & $71.92 \mathrm{Ba}$ & $40.75 \mathrm{Bb}$ & 56.35 & & & & \\
\hline Mean & 78.42 & 42.82 & & & & & \\
\hline \multicolumn{4}{|c|}{$\mathrm{NDFap}^{4}$ (g kg DM) } & 1.1364 & 0.1269 & $<0.001$ & 0.1189 \\
\hline 08:00 & 694.12 & 763.17 & $728.65 \mathrm{~A}$ & & & & \\
\hline $14: 00$ & 668.62 & 763.47 & $716.05 \mathrm{~A}$ & & & & \\
\hline Mean & $681.37 b$ & $763.32 \mathrm{a}$ & & & & & \\
\hline \multicolumn{4}{|c|}{$\mathrm{ADF}^{5}(\mathrm{~g} \mathrm{~kg} \mathrm{DM})$} & 0.7731 & 0.2659 & $<0.001$ & 0.6334 \\
\hline 08:00 & 488.00 & 543.10 & $515.55 \mathrm{~A}$ & & & & \\
\hline $14: 00$ & 480.30 & 539.95 & $510.12 \mathrm{~A}$ & & & & \\
\hline Mean & $484.15 b$ & $541.52 \mathrm{a}$ & & & & & \\
\hline \multicolumn{4}{|c|}{$\operatorname{NDIN}^{6}(\% \mathrm{TN})$} & 0.8584 & $<0.001$ & $<0.001$ & 0.1336 \\
\hline 08:00 & 11.95 & 17.44 & $14.70 \mathrm{~B}$ & & & & \\
\hline $14: 00$ & 13.83 & 20.43 & $17.13 \mathrm{~A}$ & & & & \\
\hline Mean & $12.89 \mathrm{~b}$ & $18.94 a$ & & & & & \\
\hline \multicolumn{4}{|c|}{$\operatorname{ADIN}^{7}(\% \mathrm{TN})$} & 0.6329 & $<0.001$ & $<0.001$ & 0.1011 \\
\hline 08:00 & 7.19 & 12.30 & $9.75 \mathrm{~B}$ & & & & \\
\hline $14: 00$ & 8.35 & 12.79 & $10.57 \mathrm{~A}$ & & & & \\
\hline Mean & $7.77 \mathrm{~b}$ & $12.55 \mathrm{a}$ & & & & & \\
\hline \multicolumn{4}{|c|}{$\operatorname{IVDMD}^{8}\left(\mathrm{~g} \mathrm{~kg}^{-1} \mathrm{DM}\right)$} & 1.7098 & 0.6832 & $<0.001$ & 0.8675 \\
\hline 08:00 & 597.35 & 463.35 & $532.35 \mathrm{~A}$ & & & & \\
\hline
\end{tabular}




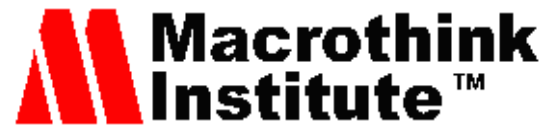

$\begin{array}{lccc}14: 00 & 600.67 & 468.75 & 534.71 \mathrm{~A} \\ \text { Mean } & 599.01 \mathrm{a} & 468.05 \mathrm{~b} & \end{array}$

† SEM, mean standard error; ${ }^{1} \mathrm{DM}$ dry matter, ${ }^{2} \mathrm{FM}$, fresh matter; ${ }^{3} \mathrm{CP}$, crude protein; ${ }^{4} \mathrm{NDF}$ ap neutral detergent fiber corrected for ash and protein, ${ }^{5} \mathrm{ADF}$, acid detergent fiber; NDIN, neutral detergent insoluble nitrogen; ${ }^{7} \mathrm{ADIN}$, acid detergent insoluble nitrogen; ${ }^{8}$ IVDMD, in vitro dry matter digestibility. Means followed by the same capital letter, in the column, and lower case, in the line, do not differ $(\mathrm{P}>0.05)$ from each other by the $\mathrm{F}$ test.

Table 5. Chemical composition, buffering capacity, $\mathrm{pH}$, and epiphytic microflora of elephant grass harvested at different cutting times (CT) and regrowth ages (RA)

\begin{tabular}{lcccc}
\hline \multirow{2}{*}{ Item } & \multicolumn{2}{c}{8 weeks } & \multicolumn{2}{c}{16 weeks } \\
\cline { 2 - 5 } & $08: 00 \mathrm{~h}$ & $14: 00 \mathrm{~h}$ & $08: 00 \mathrm{~h}$ & $14: 00 \mathrm{~h}$ \\
\hline $\mathrm{DM}^{1}\left(\mathrm{~g} \mathrm{~kg} \mathrm{FM}^{10}\right)$ & 105.3 & 119.4 & 204.0 & 215.0 \\
$\mathrm{WSC}^{2}(\mathrm{~g} \mathrm{~kg} \mathrm{DM})$ & 49.2 & 60.4 & 45.9 & 51.80 \\
$\mathrm{CP}^{3}(\mathrm{~g} \mathrm{~kg} \mathrm{DM})$ & 106.52 & 112.32 & 81.80 & 83.10 \\
$\mathrm{NDFap}^{4}(\mathrm{~g} \mathrm{~kg} \mathrm{DM})$ & 645.52 & 613,52 & 699.07 & 686.92 \\
$\mathrm{ADF}^{5}(\mathrm{~g} \mathrm{~kg} \mathrm{DM})$ & 645.52 & 613.52 & 699.07 & 686.92 \\
$\mathrm{NIDN}^{6}(\% \mathrm{TN})$ & 29.70 & 33.97 & 27.38 & 21.94 \\
$\mathrm{ADIN}^{7}(\% \mathrm{TN})$ & 7.13 & 6.95 & 8.16 & 8.20 \\
$\mathrm{BC}^{8}($ meq de NaOH$/ 100 \mathrm{~g}$ de DM$)$ & 14.36 & 15.34 & 9.25 & 9.62 \\
$\mathrm{pH}_{\mathrm{LAB}}$ & 6.41 & 6.29 & 6.04 & 6.18 \\
Enterobacter (log ufc g FM) & 7.15 & 6.14 & 6.36 & 6.13 \\
Mold and yeast (log ufc g FM). & 7.05 & 6.98 & 6.07 & 6.74 \\
\hline
\end{tabular}

${ }^{1} \mathrm{DM}$, dry matter; $\mathrm{WSC}^{2}$, water soluble carbohydrates ${ }^{2}, \mathrm{CP}^{3}$, crude protein; ${ }^{4} \mathrm{NDFap}$ neutral detergent fiber corrected for ash and protein; ${ }^{5} \mathrm{ADF}$, acid detergent fiber; ${ }^{6} \mathrm{NDIN}$, neutral detergent insoluble nitrogen; ${ }^{7} \mathrm{ADIN}$, acid detergent insoluble nitrogen; ${ }^{8} \mathrm{BC}$, buffering capacity. ${ }^{9} \mathrm{LAB}$, lactic acid bacteria. ; ${ }^{10} \mathrm{FM}$, fresh matter.

Table 6. Fermentation profile and microbial population of elephant grass silages harvested at different cutting times (CT) and regrowth ages (RA)

\begin{tabular}{|c|c|c|c|c|c|c|c|}
\hline \multirow{2}{*}{$\begin{array}{l}\text { Cutting Time } \\
\text { (CT) }\end{array}$} & \multicolumn{2}{|c|}{ Regrowth age (weeks) } & \multirow{2}{*}{ Mean } & \multirow{2}{*}{$\mathrm{SEM}^{\dagger}$} & \multicolumn{3}{|c|}{ P-value } \\
\hline & 8 & 16 & & & $\mathrm{H}$ & RA & $\mathrm{H} \times \mathrm{RA}$ \\
\hline & \multicolumn{2}{|c|}{$\mathrm{pH}$} & & 0,1356 & $<0.001$ & 0.0018 & 0.0039 \\
\hline 08:00 & $5.02 \mathrm{Aa}$ & $4.29 \mathrm{Ab}$ & 4.66 & & & & \\
\hline $14: 00$ & $3.83 \mathrm{Ba}$ & $3.79 \mathrm{Ba}$ & 3.81 & & & & \\
\hline Mean & 4.43 & 4.04 & & & & & \\
\hline \multicolumn{4}{|c|}{ Lactic acid $\left(\mathrm{g} \mathrm{kg} \mathrm{DM}^{1}\right)$} & 0.2414 & 0.0012 & $<0.001$ & 0.0034 \\
\hline 08:00 & $34.52 \mathrm{Aa}$ & $13.17 \mathrm{Bb}$ & 23.85 & & & & \\
\hline $14: 00$ & $35.40 \mathrm{Aa}$ & $25.05 \mathrm{Ab}$ & 30.22 & & & & \\
\hline Mean & 34.96 & 19.11 & & & & & \\
\hline \multicolumn{4}{|c|}{ Acetic acid (g kg DM) } & 0.2503 & $<0.001$ & $<0.001$ & $<0.001$ \\
\hline 08:00 & $28.97 \mathrm{Aa}$ & $10.17 \mathrm{Ab}$ & 19.57 & & & & \\
\hline $14: 00$ & $7.22 \mathrm{Ba}$ & $5.45 \mathrm{Ba}$ & 6.33 & & & & \\
\hline Mean & 18.10 & 7.81 & & & & & \\
\hline
\end{tabular}




\begin{tabular}{|c|c|c|c|c|c|c|c|}
\hline Q & $0 G 0 \mathrm{Ph}$ & & & & & & \\
\hline $08: 00$ & $0.60 \mathrm{Bb}$ & 3.20Aa & 1.90 & & & & \\
\hline $14: 00$ & 7.37Aa & $2.60 \mathrm{Bb}$ & 4.99 & & & & \\
\hline Mean & 3.98 & 2.90 & & & & & \\
\hline \multicolumn{4}{|c|}{ Butyric acid (g kg DM) } & 0.1368 & $<0.001$ & $<0.001$ & $<0.001$ \\
\hline 08:00 & $12.35 \mathrm{Aa}$ & $0.50 \mathrm{Ab}$ & 6.42 & & & & \\
\hline $14: 00$ & $0.63 \mathrm{Ba}$ & $0.45 \mathrm{Aa}$ & 0.54 & & & & \\
\hline Mean & 6.48 & 0.48 & & & & & \\
\hline \multicolumn{4}{|c|}{ Ethanol (g kg DM) } & 0.0992 & 0.0641 & $<0.001$ & 0.1375 \\
\hline 08:00 & 16.45 & 11.97 & $14.21 \mathrm{~A}$ & & & & \\
\hline $14: 00$ & 15.97 & 8.12 & $12.05 \mathrm{~A}$ & & & & \\
\hline Mean & $16.21 \mathrm{a}$ & $10.05 b$ & & & & & \\
\hline \multicolumn{4}{|c|}{$\mathrm{NH}_{3}-\mathrm{N}(\mathrm{g} \mathrm{kg} \mathrm{TN})$} & 3.1356 & $<0.001$ & $<0.001$ & $<0.001$ \\
\hline 08:00 & 382.1Aa & $114.8 \mathrm{Ab}$ & 248.4 & & & & \\
\hline $14: 00$ & $89.2 \mathrm{Ba}$ & $108.8 \mathrm{Aa}$ & 98.9 & & & & \\
\hline Mean & 235.6 & 111.8 & & & & & \\
\hline \multicolumn{4}{|c|}{$\mathrm{LAB}^{3}\left(\log\right.$ ufc g FM $\left.{ }^{5}\right)$} & 0.1802 & 0.5148 & 0.0038 & 0.9052 \\
\hline 08:00 & 7.45 & 8.48 & $7.96 \mathrm{~A}$ & & & & \\
\hline $14: 00$ & 7.30 & 8.26 & $7.78 \mathrm{~A}$ & & & & \\
\hline Mean & $7.38 b$ & $8.37 \mathrm{a}$ & & & & & \\
\hline \multicolumn{8}{|c|}{ Enterobacteria (log ufc g FM) } \\
\hline 08:00 & $\mathrm{ND}^{4}$ & ND & ND & - & - & - & - \\
\hline $14: 00$ & ND & ND & ND & & & & \\
\hline Mean & ND & ND & & & & & \\
\hline \multicolumn{8}{|c|}{$\begin{array}{c}\text { Mold and yeast (log ufc g } \\
\text { FM) }\end{array}$} \\
\hline 08:00 & $\mathrm{ND}^{4}$ & ND & ND & - & - & - & - \\
\hline $14: 00$ & ND & ND & ND & & & & \\
\hline Mean & ND & ND & & & & & \\
\hline \multicolumn{4}{|c|}{ Flieg's Point } & 7.0604 & $<0.001$ & $<0.001$ & 0.0052 \\
\hline 08:00 & $25.60 \mathrm{Bb}$ & $73.48 \mathrm{Ba}$ & 49.54 & & & & \\
\hline $14: 00$ & $77.23 \mathrm{Ab}$ & $97.75 \mathrm{Aa}$ & 87.49 & & & & \\
\hline Mean & 51.42 & 85.41 & & & & & \\
\hline
\end{tabular}

${ }^{\dagger} \mathrm{SEM}$, mean standard error; ${ }^{1} \mathrm{DM}$, dry matter; ${ }^{2} \mathrm{NH}_{3} / \mathrm{TN}$, ammoniacal nitrogen in relation to total nitrogen; ${ }^{3} \mathrm{LAB}$, lactic acid bacteria, ${ }^{4} \mathrm{ND}$, not detected (count less than dilution $10^{1}$ ); ${ }^{5} \mathrm{FM}$, fresh matter. Means followed by the same capital letter, in the column, and lower case, in the line, do not differ $(\mathrm{P}>0.05)$ from each other by the $\mathrm{F}$ test.

\subsubsection{Trial 2}

The results of Trial 2 are presented in Table 7. The DM and NDFap variables were significantly affected by CT $(P<0.05)$ and by RA $(P<0.05)$. Higher DM levels were observed in the silage of elephant grass harvested at 16 weeks or 14:00, while lower NDF levels were obtained for elephant grass harvested at 8 weeks or 14:00. A significant CT $\times$ RA 
interaction $(P<0.05)$ effect was observed on CP, ADF, NDIN, and ADIN and IVDMD coefficients. The CP contents were higher, and ADF and ADIN contents lower in the silage of plants harvested at 14:00 than at 08:00 at both RAs. Thus, the IVDMD coefficients were higher in plant silages harvested at 14:00 than at 08:00 at both RAs.

Table 7. Nutritive value of elephant grass silages harvested at different cutting times (CT) and regrowth ages (RA)

\begin{tabular}{|c|c|c|c|c|c|c|c|}
\hline \multirow{2}{*}{$\begin{array}{l}\text { Cutting Time } \\
\text { (CT) }\end{array}$} & \multicolumn{2}{|c|}{ Regrowth age (weeks) } & \multirow{2}{*}{ Mean } & \multirow{2}{*}{$\mathrm{SEM}^{\dagger}$} & \multicolumn{3}{|c|}{ P-value } \\
\hline & 8 & 16 & & & $\mathrm{H}$ & RA & $\mathrm{H} \times \mathrm{RA}$ \\
\hline \multicolumn{4}{|c|}{$\mathrm{DM}^{1}\left(\mathrm{~g} \mathrm{~kg} \mathrm{FM}^{2}\right)$} & 1.2432 & $<0.001$ & $<0.001$ & 0.9114 \\
\hline 08:00 & 108.5 & 201.9 & 155.2B & & & & \\
\hline $14: 00$ & 127.6 & 221.7 & $174.7 \mathrm{~A}$ & & & & \\
\hline Mean & $118.8 \mathrm{~B}$ & $211.8 \mathrm{~A}$ & & & & & \\
\hline \multicolumn{4}{|c|}{$\mathrm{CP}^{3}(\mathrm{~g} \mathrm{~kg} \mathrm{DM})$} & 0.5737 & $<0.001$ & $<0.001$ & $<0.001$ \\
\hline 08:00 & $54.75 \mathrm{Bb}$ & $83.60 \mathrm{Ba}$ & 69.17 & & & & \\
\hline $14: 00$ & $115.30 \mathrm{Aa}$ & $98.00 \mathrm{Ab}$ & 106.65 & & & & \\
\hline Mean & 85.02 & 90.80 & & & & & \\
\hline \multicolumn{4}{|c|}{$\operatorname{NDFap}^{4}(\mathrm{~g} \mathrm{~kg} \mathrm{DM})$} & 1.0307 & $<0.001$ & $<0.001$ & 0.1191 \\
\hline 08:00 & 698.15 & 730.45 & $714.30 \mathrm{~A}$ & & & & \\
\hline $14: 00$ & 625.62 & 681.72 & $653.67 \mathrm{~B}$ & & & & \\
\hline Mean & $661.88 B$ & $706.08 \mathrm{~A}$ & & & & & \\
\hline \multicolumn{4}{|c|}{$\mathrm{ADF}^{5}(\mathrm{~g} \mathrm{~kg} \mathrm{DM})$} & 0.6967 & $<0.001$ & 0.3362 & 0.0239 \\
\hline 08:00 & $515.02 \mathrm{Aa}$ & $505.05 \mathrm{Aa}$ & 510.03 & & & & \\
\hline $14: 00$ & $452.57 \mathrm{Bb}$ & $475.17 \mathrm{Ba}$ & 463.87 & & & & \\
\hline Mean & 483.80 & 490.11 & & & & & \\
\hline \multicolumn{4}{|c|}{$\operatorname{NDIN}^{6}(\% \mathrm{TN})$} & 1.6789 & $<0.001$ & $<0.001$ & $<0.001$ \\
\hline 08:00 & 26.08Aa & $12.22 \mathrm{Ab}$ & 19.15 & & & & \\
\hline $14: 00$ & $10.06 \mathrm{Bb}$ & $11.48 \mathrm{Aa}$ & 10.75 & & & & \\
\hline Mean & 18.07 & 11.85 & & & & & \\
\hline \multicolumn{4}{|c|}{$\operatorname{ADIN}^{7}(\% \mathrm{TN})$} & 1.0094 & $<0.001$ & $<0.001$ & $<0.001$ \\
\hline 08:00 & 7.27Ab & $15.55 \mathrm{Aa}$ & 11.41 & & & & \\
\hline $14: 00$ & $5.80 \mathrm{Bb}$ & 7.07Ba & 6.44 & & & & \\
\hline Mean & 6.54 & 11.31 & & & & & \\
\hline \multicolumn{4}{|c|}{ IVDMD $^{8}($ g kg DM) } & 1.3351 & $<0.001$ & $<0.001$ & 0.0006 \\
\hline 08:00 & $539.32 \mathrm{Ba}$ & $526.17 \mathrm{Ba}$ & 532.75 & & & & \\
\hline $14: 00$ & $653.15 \mathrm{Aa}$ & $562.27 \mathrm{Ab}$ & 607.71 & & & & \\
\hline Mean & 596.23 & 544.22 & & & & & \\
\hline
\end{tabular}

${ }^{\dagger}$ SEM, mean standard error; ${ }^{1} \mathrm{DM}$, dry matter, ${ }^{2} \mathrm{FM}$, fresh matter; ${ }^{3} \mathrm{CP}$, crude protein; ${ }^{4} \mathrm{NDFap}$ neutral detergent fiber corrected for ash and protein; ${ }^{5} \mathrm{ADF}$, acid detergent fiber; ${ }^{6} \mathrm{NDIN}$, neutral detergent insoluble nitrogen; ${ }^{7} \mathrm{ADIN}$, acid detergent insoluble nitrogen; ${ }^{8} \mathrm{IVDMD}$, in vitro dry matter digestibility. Means followed by the same capital letter, in the column, and lower case, in the line, do not differ $(\mathrm{P}>0.05)$ from each other by the $\mathrm{F}$ test. 


\section{Discussion}

The initial concentration of soluble carbohydrates in the plant at the time of ensiling affects the organic acid production, since that are used as a substrate by LAB. Lactic acid causes the rapid decline in $\mathrm{pH}$ and maintains forage stability during storage in the silo by inhibiting or eliminating undesirable and low pH-intolerant microorganisms (Kung Jr. et al., 2018). As verified in the present study, in both trials, the highest concentration of soluble carbohydrates in elephant grass harvested at 14:00 was responsible for increasing lactic acid levels and reducing silage $\mathrm{pH}$, consequently improving silage quality (Tables 2, 3, 5, and 6).

The silage from elephant grass harvested at 08:00, at 8 weeks, showed satisfactory lactic acid concentrations (34.52 $\mathrm{g} \mathrm{kg}^{-1} \mathrm{DM}$; Trial 2) according to Kung Jr. et al. (2018), who reported lactic acid concentrations of 20 to $40 \mathrm{~g} \mathrm{~kg}^{-1} \mathrm{DM}$ for well-fermented silages. However, these silages showed higher $\mathrm{pH}$ values (5.02) relative to the other treatments, which may be related to the high levels of butyric and acetic acids, and ammonia $\left(\mathrm{NH}_{3}-\mathrm{TN}\right.$; Table 3). These compounds produced through fermentation of soluble carbohydrates and protein degradation by clostridia and enterobacteria increase the buffering capacity of the forage in the silo, preventing $\mathrm{pH}$ from declining (Dong et al., 2018).

The lower $\mathrm{pH}$ values in silages produced from elephant grass harvested at 14:00 relative to those of silages produced from elephant grass harvested at 08:00 (Trial 1) corroborate the results presented by Guo et al. (2015), who observed a $\mathrm{pH}$ value of 4.07 in the silages produced from elephant grass harvested at 08:00, and 3.87 in silage produced from elephant grass harvested at 13:00. The same authors reported an increase in the concentration of soluble carbohydrates, from 80.6 to $107 \mathrm{~g} \mathrm{~kg}^{-1} \mathrm{DM}$ in elephant grass harvested in the morning and afternoon, respectively.

The levels of acetic acid in both trials (except for silages produced with 8 week plants at 08:00 in Trial 2) were less than $10 \mathrm{~g} \mathrm{~kg}^{-1} \mathrm{DM}$, below the limits recommended by Kung Jr. et al. (2018) (10-30 $\left.\mathrm{g} \mathrm{kg}^{-1} \mathrm{MS}\right)$ and Gerlach et al. (2021) $\left(17 \mathrm{~g} \mathrm{~kg}^{-1} \mathrm{MS}\right)$ that are characteristic of well-fermented silage. The butyric acid levels observed in the trial 1 silage were considered adequate, consistent with Kung Jr. et al. (2018) at $<1 \mathrm{~g} \mathrm{~kg}^{-1} \mathrm{DM}$ (Table 3). However, in trial 2, silages from plants harvested at 8 weeks, at 08:00 am, had a high butyric acid concentration $\left(12.35 \mathrm{~g} \mathrm{~kg}^{-1} \mathrm{DM}\right)$, which can be explained by the occurrence of secondary fermentation mainly because of bacteria of the genus Clostridium (Driehuis et al., 2018). Clostridium-fermented silages, in addition to the presence of butyric acid, have a high $\mathrm{pH}$ and high concentrations of acetic acid and $\mathrm{NH}_{3}-\mathrm{TN}$ (Oladosu et al., 2016; Kung Jr. et al., 2018) (Table 6). 
Generally, silages from plants harvested with a low DM content have higher soluble $\mathrm{N}$ and ammoniacal nitrogen concentrations than silages with a high DM content. In the present study, although the elephant grass DM values were below the recommended (25-35\%), the silage (except at 8 weeks, at 08:00 in Trial 2) presented low NH3-TN concentrations $\left(<12 \mathrm{~g} \mathrm{~kg}^{-1}\right.$ DM), which indicates good fermentation quality, according to the criteria proposed by Kung Jr. et al. (2018). The lower levels of $\mathrm{NH}_{3}-\mathrm{TN}$ could be attributed to the low proteolytic activity of microorganisms, such as bacteria of the genus Clostridium, which are inhibited because of the rapid decline in $\mathrm{pH}$ during the fermentation of soluble carbohydrates by the LAB epiphytic population. (Ni et al., 2017; Wang et al., 2019a).

The epiphytic LAB population present in forage is important for silage fermentation. For good forage fermentation, the required LAB population is $5 \log \mathrm{CFU} \mathrm{g}^{-1}$ fresh weight (FW) (Pahlow et al., 2003). LAB populations $>5 \log \mathrm{CFU} \mathrm{g}{ }^{-1} \mathrm{FW}$ were observed in both trials, which benefited the fermentation process during ensiling (Tables 2 and 5). The variation in the epiphytic LAB population in elephant grass between CT and RA is probably associated with environmental conditions, such as humidity, temperature, and solar radiation (Guo et al., 2015). These authors did not observe differences in the LAB population in elephant grass silages produced from plants harvested at 08:00 and 13:00. In the present study, this behavior was observed only in trial 2 (Table 6), while in trial 1, there was an increase in the LAB population in silage produced from plants harvested at 8 weeks at 14:00. As seen in literature and the present work, further studies are needed to understand the behavior of LAB in silages produced with forages harvested at different times.

The low mold count in the silages of the two trials is probably related to the high mass compaction at the time of ensiling (141-182 $\left.\mathrm{kg} \mathrm{m}^{-3} \mathrm{DM}\right)$, which favored rapid oxygen exhaustion inside the silos. In addition to compaction, acetic acid fermentation may have also contributed to reducing the fungal population since acetic acid has antifungal properties (Kung Jr. et al., 2018; Muck et al., 2018). Molds and yeasts are the main ethanol-producing microorganisms during the fermentation period in the silo (Kung Jr. et al., 2018). Despite the low mold and yeast populations observed, ethanol concentrations in the silage produced in trial 2 (mainly after 8 weeks of regrowth, $16.21 \mathrm{~g} \mathrm{~kg}^{-1} \mathrm{DM}$ ) were higher than those in trial 1 (Tables 3 and 6). Some authors have reported that in addition to that produced from molds and yeasts, ethanol can be produced by enterobacteria, and heterolactic bacteria (Gomes et al., 2019; Wang et al., 2020; Ávila and Carvalho, 2020). This could explain the higher ethanol production observed in the silages in the present study, which was above the level suitable for tropical grass silages (5-10 $\mathrm{g} \mathrm{kg}^{-1} \mathrm{DM}$ ) according to Kung Jr. et al. (2018).

Based on Flieg's point evaluation system, the silage fermentation in Trial 1 was classified as very good, indicating silage of adequate quality since all silages presented a Flieg's point $>80$. In Trial 2, the quality of the silage produced with elephant grass harvested at 14:00 and 16 weeks was classified as having very good fermentation quality; silages produced with elephant grass harvested at 8 weeks at 14:00 and 16 weeks at 08:00 were considered good quality silages. Those produced with elephant grass harvested at 8 weeks at 08:00 were considered to be of poor quality. 
Flieg's point, based on the DM content and the $\mathrm{pH}$ value of silages, has been used to evaluate silage quality, although there are some limitations in this scoring system. According to Yuan et al. (2017), Flieg's point does not consider organic acids, the extent of protein degradation, or the aerobic stability of the silage.

For good silage fermentation, it is recommended to cut grasses with a DM content of 25-35\% (Kung Jr et al., 2018). Although the DM content of plants harvested at 8 weeks was below the minimum requirement, the results obtained in the present study showed that it was possible to produce silages of adequate nutritional value (Tables 4 and 7). In a study with elephant grass cv Napier, De Oliveira et al. (2014) observed DM content of $20.5 \%$ and $16.8 \%$ when the cut was made in the afternoon and in the morning, respectively. In a similar study, De Oliveira et al. (2018) also observed an increase in DM accumulation in Marandu palisade grass from 06:00 to 15:00. Guo et al. (2015) reported increased DM concentrations in plants and silages produced from elephant grass harvested in the afternoon (13:00) than at 8:00, as verified in the present study. The higher DM values observed in the silages (Tables 4 and 7) relative to the elephant grass at the harvest time (Tables 2 and 5) could be attributed to the effluent losses observed after opening the experimental silos; however, this was not quantified in the present study.

The reduction of concentrations of soluble carbohydrates in the plant increases the concentration of CP (De Oliveira et al., 2014; Wang et al., 2020a), which explains the greater attributions of this nutrient in the silages produced in the morning than from silages produced in the afternoon (Tables 2,4,5, and 7). De Oliveira et al. (2014) reported an 85\% increase in soluble carbohydrates and a 12\% reduction in $\mathrm{N}$ from 06:00 to 18:00 in Cenchrus purpureus cv. Napier. De Oliveira et al. (2018), who evaluated the diurnal vertical and seasonal variations of non-structural carbohydrates in Marandu grass, observed a similar result. In trial 2 , in the silage produced at 08:00 h at 8 weeks, significant reduction in CP and increase in $\mathrm{N}-\mathrm{NH} 3$ contents, butyric and acetic acids (Table 6) indicate the occurrence of proteolysis in the silo, which is related to clostridial and/or enterobacterial activity (Wang et al., 2020), especially under conditions of high humidity.

Since nitrogen compounds are present in the cellular content of young plants, with increasing $\mathrm{RA}$, there is a reduction or dilution of the CP content due to the accumulation of structural carbohydrates. Monção et al. (2019) verified a linear reduction of $0.37 \mathrm{~g} \mathrm{~kg}^{-1}$ per day in the $\mathrm{CP}$ concentration in BRS Capiaçu, with an average of $123,2 \mathrm{~g} \mathrm{~kg}^{-1}$ in 30 days. A similar effect was observed in the present study since silages of elephant grass harvested at 16 weeks presented lower $\mathrm{CP}$ concentrations than silages of elephant grass harvested at 8 weeks. When evaluating chemical composition, before and after ensiling of elephant grass BRS Capiaçu at four regrowth ages (50, 70, 90, and 110 days), Lopes et al. (2021) verified linear decreases in $\mathrm{CP}$ content with an increase in RA.

In Trial 1, silage of elephant grass harvested at 16 weeks showed lower IVDMD coefficients, probably because of the increase in the NDF and ADF levels that occurred with advancing RA. A similar result was observed by Lopes et al. (2021), with the advancing RA of elephant grass cv. BRS Capiaçu. In Trial 2, the highest IVDMD coefficients in elephant grass silage harvested at 14:00 could be attributed to the lower levels of NDFap, ADF, NDIN, and ADIN. 
A similar result was observed by De Oliveira et al. (2019) in a study with palisade grass harvested in the afternoon compared with that collected in the morning.

Earlier RA and later cutting of elephant grass improved the chemical composition of the grass and the fermentation process in the silo. Therefore, harvesting elephant grass at 8 weeks and 14:00 is recommended to produce better quality silage without wilting or additives. Additional studies are needed to evaluate the intake and performance of animals fed the tested silages.

\section{References}

Ávila, C. L. S., \& Carvalho, B. F. (2020). Silage fermentation updates focusing on the performance of microorganisms. Journal of Applied Microbiology, 128(4), 966-984. https://doi.org/10.1111/jam.14450

Bernardes, T. F., Daniel, J. L. P., Adesogan, A. T., McAllister, T. A., Drouin, P., Nussio, L. G. Huhtanen, G. F. Tremblay, G. Bélanger, \& Cai, Y. (2018). Silage review: Unique challenges of silages made in hot and cold regions. Journal of Dairy Science, 101(5), 4001-4019. https://doi.org/10.3168/jds.2017-13703

De Oliveira, F. L., Sanchez, J. M. D., Vendramini, J. M. B., Lima, C. G., Luz, P. H. C., Rocha, C. O., ... Herling, V. R. (2018). Diurnal vertical and seasonal changes in non-structural carbohydrates in Marandu palisade grass. Journal of Agricultural Science, 156, 457-464. https://doi.org/10.1017/S0021859618000394

De Oliveira, L. P., Paiva, A. J., Pereira, L. E., Geremia, E. V., \& Da Silva, S. C. (2014). Morning and afternoon sampling and herbage chemical composition of rotationally stocked elephant grass cv. Napier. Tropical Grasslands-Forrajes Tropicales, 2(1), 106-107. https://doi.org/10.17138/TGFT(2)106-107

Detmann, E., Souza, M. A., Valadares Filho, S. C., Queiroz, A. C., Berchielli, T. T., Saliba, E. O. S., ... Azevedo, J. A. G. (2012). Métodos para Análise de Alimentos. Visconde do Rio Branco, MG: Suprema, 214p.

Dong, Z., Chen, L., Li, J., Yuan, X., \& Shao, T. (2019). Characterization of nitrogen transformation dynamics in alfalfa and red clover and their mixture silages. Grassland science, 65(2), 109-115. https://doi.org/10.1111/grs.12230

Driehuis, F., Wilkinson, J. M., Jiang, Y., Ogunade, I., \& Adesogan, A. T. (2018). Silage review: animal and human health risks from silage. Journal of Dairy Science, 101(5), 4093-4110. https://doi.org/10.3168/jds.2017-13836

Gerlach, K., Daniel, J. L. P., Jobim, C. C., \& Nussio, L. G. (2021). A data analysis on the effect of acetic acid on dry matter intake in dairy cattle. Animal Feed Science and Technology, 272, 114782. https://doi.org/10.1016/j.anifeedsci.2020.114782

Gomes, A. L. M., Jacovaci, F. A., Bolson, D. C., Nussio, L. G., Jobim, C. C., \& Daniel, J. L. P. (2019). Effects of light wilting and heterolactic inoculant on the formation of volatile organic compounds, fermentative losses and aerobic stability of oat silage. Animal Feed Science and 
Technology, 247, 194-198. https://doi.org/10.1016/j.anifeedsci.2018.11.016

Guo, G., Yu, C., Wang, Q., Xin, P., Shimojo, M., \& Shao, T. (2014). Silage fermentation characteristics of Italian ryegrass (Lolium multiflorum Lam.) harvested at various times on a sunny day. Crop Science, 54(2), 851-858. https://doi.org/10.2135/cropsci2012.12.0692

Guo, G., Yuan, X., Wen, A., Liu, Q., Zhang, S., \& Shao, T. (2015). Silage fermentation characteristics of napiergrass harvested at various times on a sunny day. Crop science, 55(1), 458-464. https://doi.org/10.2135/cropsci2014.03.0172

Haryani, H., Norlindawati, A. P., Norfadzrin, F., Aswanimiyuni, A., \& Azman, A. (2018). Yield and nutritive values of six Napier (Pennisetum purpureum) cultivars at different cutting age. Malaysian Journal of Veterinary Research, 9(2), 6-12. https://doi.org/10.5251/abjna.2016.7.5.213.219

Holden, L. A. (1999). Comparison of methods of in vitro dry matter digestibility for ten feeds. Journal of Dairy $\quad$ Science, 82(8), $\quad$ 1791-1794. https://doi.org/10.3168/jds.S0022-0302(99)75409-3

Instituto Nacional de Meteorologia do Brasil - INMET. Normais Climatológicas. Brasília DF, 2016. Available in: http://www.inmet.gov.br/portal/.

Kaiser, E., K. Weib, L.V. Polip. (2002). A new concept for the estimation of the ensiling potential of forages. In: International Grassland Congress, 13, Proceedings... Auchincruive, Scotland, p. 344-358.

Köppen, W., \& Geiger, R. (Eds.). (1930). Handbuch der klimatologie (Vol. 1). Berlin: Gebrüder Borntraeger.

Kung Jr., L., Shaver, R. D., Grant, R. J., \& Schmidt, R. J. (2018). Silage review: Interpretation of chemical, microbial, and organoleptic components of silages. Journal of Dairy Science, 101(5), 4020-4033. https://doi.org/10.3168/jds.2017-13909

Kung Jr., L., Taylor, C. C., Lynch, M. P., \& Neylon, J. M. (2003). The effect of treating alfalfa with Lactobacillus buchneri 40788 on silage fermentation, aerobic stability, and nutritive value for lactating dairy cows. Journal of Dairy Science,86(1), 336-343. https://doi.org/10.3168/jds.S0022-0302(03)73611-X

Kung, L., Jr. (1996). Preparation of silage water extracts for chemical analyses: standard operating procedures. Worrilow: University of Delaware, Ruminat Nutrition Laboratory.

Lopes, F. C. F., Morenz, M. J. F., da Silva Lédo, F. J., da Costa Carneiro, J., Paciullo, D. S. C., Andrade, P. J. M., \& de Moraes, C. T. (2021). Chemical composition and fatty acid profile of BRS Capiaçu ensiled at different regrowth ages. Semina: Ciências Agrárias, 42(3Supl1), 1981-2004. https://doi.org/10.5433/1679-0359.2021v42n3Supl1p1981

Monção, F. P., Costa, M. A. M. S., Rigueria, J. P. S., Moura, M. M. A., Júnior, V. R. R., Gomes, V. M., ... Chamone, J. M. A. (2019). Yield and nutritional value of BRS Capiaçu grass at different regrowth ages. Semina: Ciências Agrárias, 40(5), 2045-2056. 
https://doi.org/10.5433/1679-0359.2019v40n5p2045

Moselhy, M. A., Borba, J. P., \& Borba, A. E. (2015). Improving the nutritive value, in vitro digestibility and aerobic stability of Hedychium gardnerianum silage through application of additives at ensiling time. Animal Feed Science and Technology, 206, 8-18. https://doi.org/10.1016/j.anifeedsci.2015.05.001

Muck, R. E., Nadeau, E. M. G., McAllister, T. A., Contreras-Govea, F. E., Santos, M. C., \& Kung Jr., L. (2018). Silage review: Recent advances and future uses of silage additives. Journal of Dairy Science, 101(5), 3980-4000. https://doi.org/10.3168/jds.2017-13839

Nelson, N. (1944). A photometric adaptation of the Somogyi method for the determination of glucose. Journal of Biological Chemistry, 153(2), 375-380. https://doi.org/10.1016/S0021-9258(18)71980-7

Ni, K., Wang, F., Zhu, B., Yang, J., Zhou, G., Pan, Y. I., ... \& Zhong, J. (2017). Effects of lactic acid bacteria and molasses additives on the microbial community and fermentation quality of soybean silage. Bioresource Technology, 238, 706-715. https://doi.org/10.1016/j.biortech.2017.04.055

Okuda, H., Fujii, S., \& Kawashima, Y. (1965). A direct colorimetric determination of blood ammonia. Tokushima Journal of Experimental Medicine, 12, 11-23.

Oladosu, Y., Rafii, M. Y., Abdullah, N., Magaji, U., Hussin, G., Ramli, A., \& Miah, G. (2016). Fermentation quality and additives: a case of rice straw silage. BioMed Research International, 2016. https://doi.org/10.1155/2016/7985167

Pahlow, G., Muck, R. E., Driehuis, F., Elferink, S. J. O., \& Spoelstra, S. F. (2003). Microbiology of ensiling. Silage Science and Technology, 42, 31-93. https://doi.org/10.2134/agronmonogr42.c2

Pereira, A. V., Lédo, F. J. D. S., \& Machado, J. C. (2017). BRS Kurumi and BRS Capiaçu-New elephant grass cultivars for grazing and cut-and-carry system. Crop Breeding and Applied Biotechnology, 17(1), 59-62. https://doi.org/10.1590/1984-70332017v17n1c9

Pholsen, S., Khota, W., Pang, H., Higgs, D., \& Cai, Y. (2016). Characterization and application of lactic acid bacteria for tropical silage preparation. Animal Science Journal, 87(10), 1202-1211. https://doi.org/10.1111/asj.12534

Playne, M. J., \& McDonald, P. (1966). The buffering constituents of herbage and of silage. Journal of the Science of Food and Agriculture, 17(6), 264-268. https://doi.org/10.1002/jsfa.2740170609

Santos, H. G., Jacomine, P. K. T., Dos Anjos, L. H. C., De Oliveira, V. A., Lumbreras, J. F., Coelho, M. R., ... Cunha, T. J. F. (2018). Sistema Brasileiro de Classificação de Solos. Brasília, DF: Embrapa, 2018.

SAS. (2016). Statistical Analysis System Institute. SAS/STAT. User's guide, version 9.4. Cary, 
North Carolina, v.2, 842 p.

Siegfried, R., Ruckemann, H., \& Stumpf, G. (1984). Method for the determination of organic-acids in silage by high-performance liquid-chromatography. Landwirtschaftliche Forschung, 37(3-4), 298-304.

Silva, L. H., Brito, A. F., Lafrenière, C., \& Berthiaume, R. (2020). Effects of cutting time and maceration on preference and nitrogen balance in beef steers fed mixed birdsfoot trefoil-timothy grass hay cut at sunrise or sundown. Translational Animal Science, 4(3), txaa168. https://doi.org/10.1093/tas/txaa168

Tremblay, G. F., Morin, C., Bélanger, G., Bertrand, A., Castonguay, Y., Berthiaume, R., \& Allard, G. (2014). Silage Fermentation of PM-and AM-Cut Alfalfa Wilted in Wide and Narrow Swaths. Crop Science, 54(1), 439-452. https://doi.org/10.2135/cropsci2013.07.0443

Wang, C., Hou, F., Wanapat, M., Yan, T., Kim, E. J., \& Scollan, N. D. (2020a). Assessment of cutting time on nutrient values, in vitro fermentation and methane production among three ryegrass cultivars. Asian-Australasian Journal of Animal Sciences, 33(8), 1242.

https://doi.org/10.5713/ajas.19.0369

Wang, S., Li, J., Dong, Z., Chen, L., \& Shao, T. (2019a). Effect of microbial inoculants on the fermentation characteristics, nutritive value, and in vitro digestibility of various forages. Animal Science Journal, 90(2), 178-188. https://doi.org/10.1111/asj.13134

Wang, S., Zhao, J., Dong, Z., Li, J., Kaka, N. A., \& Shao, T. (2020). Sequencing and microbiota transplantation to determine the role of microbiota on the fermentation type of oat silage. Bioresource Technology, 309, 123371. https://doi.org/10.1016/j.biortech.2020.123371

Wang, Y., Chen, X., Wang, C., He, L., Zhou, W., Yang, F., \& Zhang, Q. (2019). The bacterial community and fermentation quality of mulberry (Morus alba) leaf silage with or without Lactobacillus casei and sucrose.Bioresource Technology, 293, 122059. https://doi.org/10.1016/j.biortech.2019.122059

Yuan, X., Wen, A., Dong, Z., Desta, S. T., \& Shao, T. (2017). Effects of formic acid and potassium diformate on the fermentation quality, chemical composition and aerobic stability of alfalfa silage. Grass and Forage Science, 72(4), 833-839. https://doi.org/10.1111/gfs.12296

Zailan, M. Z., Yaakub, H., \& Jusoh, S. (2016). Yield and nutritive value of four Napier (Pennisetum purpureum) cultivars at different harvesting ages. Agriculture and Biology Journal of North America., 7, 213-9. https://doi.org/10.5433/1679-0359.2019v40n5p2045

\section{Copyright Disclaimer}

Copyright for this article is retained by the author(s), with first publication rights granted to the journal.

This is an open-access article distributed under the terms and conditions of the Creative Commons Attribution license (http://creativecommons.org/licenses/by/4.0/). 